\title{
Surface enhanced vibrational spectroscopy using ultra narrow-band perfect absorber
}

\author{
Aksu, Serap
}

Serap Aksu, "Surface enhanced vibrational spectroscopy using ultra narrowband perfect absorber," Proc. SPIE 11460, Metamaterials, Metadevices, and Metasystems 2020, 114601W (20 August 2020); doi: 10.1117/12.2568449

SPIE. Event: SPIE Nanoscience + Engineering, 2020, Online Only 


\title{
Surface enhanced vibrational spectroscopy using ultra narrow-band perfect absorber
}

\author{
Serap Aksu \\ Koc University, Dept. of Physics, Istanbul, Turkey
}

\begin{abstract}
In this work, we present a narrow-band (sub-100 $\mathrm{cm}^{-1}$ ) and multi-band metamaterial perfect absorber operating in the mid-IR frequency regime for analysis of biomolecules with vibrational spectroscopy. The presented perfect absorber is based on multi-layer metamaterial plasmonic nanoantennas. We used numerical and experimental work to fine tune the parameters of the PA and control its spectral response. The suggested PA system provides high absorbance values $(100 \%)$ in the mid-IR region and near-field enhancement factor of $10^{3}$ at the corresponding resonance values. The experimental results are well agreeing the numerical results. The working principle of the presented perfect absorption can be both explained by impedance matching and critical coupling phenomenon. The fabricated PA shows strong narrow band resonance and all the resonance energy could be transferred to thin films $(10 \mathrm{~nm})$ for higher sensitivity. In addition, PA shows multi-band resonances within the mid-IR region, thus it can be effectively used for simultaneously detecting the different biomolecular fingerprints. In this sense, we experimentally observed absorption of carbonyl $(\mathrm{C}=\mathrm{O})$ and asymmetric methyl $\left(-\mathrm{CH}_{3}\right)$ stretching bands of thin polymethyl methacrylate (PMMA) film on the narrow band resonance of PA.
\end{abstract}

Keywords: Narrow-band perfect absorber, metamaterial, surface enhanced IR absorption spectroscopy, multi-band spectroscopy.

\section{INTRODUCTION}

Metamaterial perfect absorbers (PAs) opened new doors to the energy harvesting devices ${ }^{1}$ and many others ${ }^{2-4}$. By providing narrow band plasmonic resonances, PAs have the potential to further improve the surface enhanced vibrational spectroscopy platforms ${ }^{5}$. Among many optical biosensing techniques vibrational spectroscopy has been instrumental as it is capable of both identification and detection of molecules. Since molecule-specific vibrations occur in mid-infrared (IR) range, light is absorbed by the dipolar molecular bond at certain frequencies, and this absorption can be tracked to identify the molecules and molecular structures/orientations ${ }^{5}$. Surface enhanced infrared absorption (SEIRA) spectroscopy technique utilizes this approach for sensitive characterization of even few biomolecules ${ }^{6}$. Surface enhancement is provided by active plasmonic surfaces that act as an antenna which can direct the light to a biomolecule (lipid, protein, polymer, etc.) at its specific absorption frequency. Thus, the energy radiated by the nanoantennas could be transferred to the biomolecule for enhancing the absorption signal intrinsic to molecular bonds ${ }^{5-6}$. The intensity of the directed light, also called the enhanced near electric field (E), is proportional to the absorption signal that arises from the biomolecule ${ }^{7-8}$. This implies that when the resonance band of the plasmonic nanoantenna is narrow, we could transfer resonance energy towards the biomolecule more effectively, precisely at the absorption frequency of the molecular bonds $^{8}$. That results in more significant absorption signal generation from the same number of molecules, thus higher sensitivity can be obtained by a narrow band resonance nanoantenna. In addition, using narrow band plasmonic resonances, we can obtain resolutions that can probe each specific molecular absorption frequencies, even if they are separated only by $100 \mathrm{~cm}^{-1}$.

\subsection{Structure Design and Numerical Analysis}

PAs can be typically designed using three functional layers that comprise a dielectric interlayer sandwiched between the bottom metal film and a patterned top metal plasmonic nanoantenna serving as a resonator ${ }^{9}$. Dielectric layer thickness allows to bring the absorption level to unity (100\%) and width of the resonance down to $85 \mathrm{~cm}^{-1}$. The top layer of PAs which are subwavelength plasmonic antenna components, absorb most of the incident radiation at the operating frequency. In earlier reports of metamaterial PAs, near unity absorbance was explained over a narrow frequency range by minimizing the reflectance with perfect impedance matching $\left(\mathrm{z}=(\mu / \varepsilon)^{1 / 2}=1\right.$, complex electric permittivity $(\varepsilon)$ and magnetic permeability $(\mu)$ ) while eliminating the transmittance with the metal film ${ }^{9}$. Another scenario to explain the 
perfect absorption is to count the radiative and intrinsic damping rates of the system which becomes equal at the resonant frequencies resulting in the critical coupling phenomenon ${ }^{3}$. Engineered PAs can create intense confined hotspots of the incident light which provide strong interaction with adjacent analytes and make them ideal candidates for biosensing and spectroscopy applications ${ }^{2,10}$. Resonance frequencies of these structures can be tuned to molecular resonance frequencies of biomolecules and polymers in mid-IR. Functionalization of PAs with molecules results in coupling plasmonic resonances with molecular absorption ${ }^{11}$. This coupling supports Fano line shapes that occur in the presence of interference between one spectrally broad and one narrow energy state ${ }^{12}$. Molecular bands of polymers have narrow band infrared spectral features ${ }^{13}$. By designing narrow band metasurfaces, we can reach these molecular bands precisely one by one.

\subsection{Materials and methods}

The PA consists of an array of combined plasmonic rods separated from an optically thick non-transparent metallic ground plate. In the optimum configuration, we use a $100 \mathrm{~nm}$-thick $\mathrm{MgF}_{2}$ spacer between a patterned $80 \mathrm{~nm}$-thick $\mathrm{Au}$ nanoparticle-based top layer and a $200 \mathrm{~nm}$-thick Au ground plate. We should note that the spacer thickness has the critical importance to achieve the unity absorption. The whole sensing platform is designed on a silicon substrate with a thickness of $500 \mathrm{~nm}$. Polarization of the illumination source is along the $\mathrm{x}$-direction. The frequency-dependent dielectric constants of the metals are taken from Palik ${ }^{14}$, and optical constants of $\mathrm{MgF}_{2}$ dielectric spacer are taken from Dodge ${ }^{15}$. We determine lengths of rods as $L 1=2600 \mathrm{~nm}, \mathrm{~L} 2=2400 \mathrm{~nm}, \mathrm{~L} 3=2200 \mathrm{~nm}, \mathrm{~L} 4=2000 \mathrm{~nm}, \mathrm{~L} 5=840 \mathrm{~nm}$, w1 $=400 \mathrm{~nm}$, $\mathrm{w} 2=200 \mathrm{~nm}$, and the array periodicities $(\mathrm{Px}=\mathrm{Py})$ are $3.2 \mu \mathrm{m}$ (Figure 1). We fabricate the PAs via electron-beam lithography (EBL) and a following lift-off process. Firstly, the $200 \mathrm{~nm}$-thick Au film and $100 \mathrm{~nm}$-thick $\mathrm{MgF}_{2}$ dielectric spacer are deposited onto the Si substrate by using electron-beam evaporation. Secondly, the $200 \mathrm{~nm}$-thick PMMA is coated as a positive photoresist on top of the $\mathrm{MgF}_{2}$ layer by spin-coating. EBL is performed on PMMA. EBL-exposed parts are then covered with 5nm-thick titanium (Ti) as an adhesion layer and $80 \mathrm{~nm}$-thick Au bye-beam evaporation, respectively. Finally, the standard lift-off process is applied to obtain nanoantennas. Fabricated PA samples are characterized optically by a commercial Bruker Hyperion 2000 FTIR microscope that has a 15x Cassegrain objective $(\mathrm{NA}=0.4)$ to collect reflection signals during the measurements.
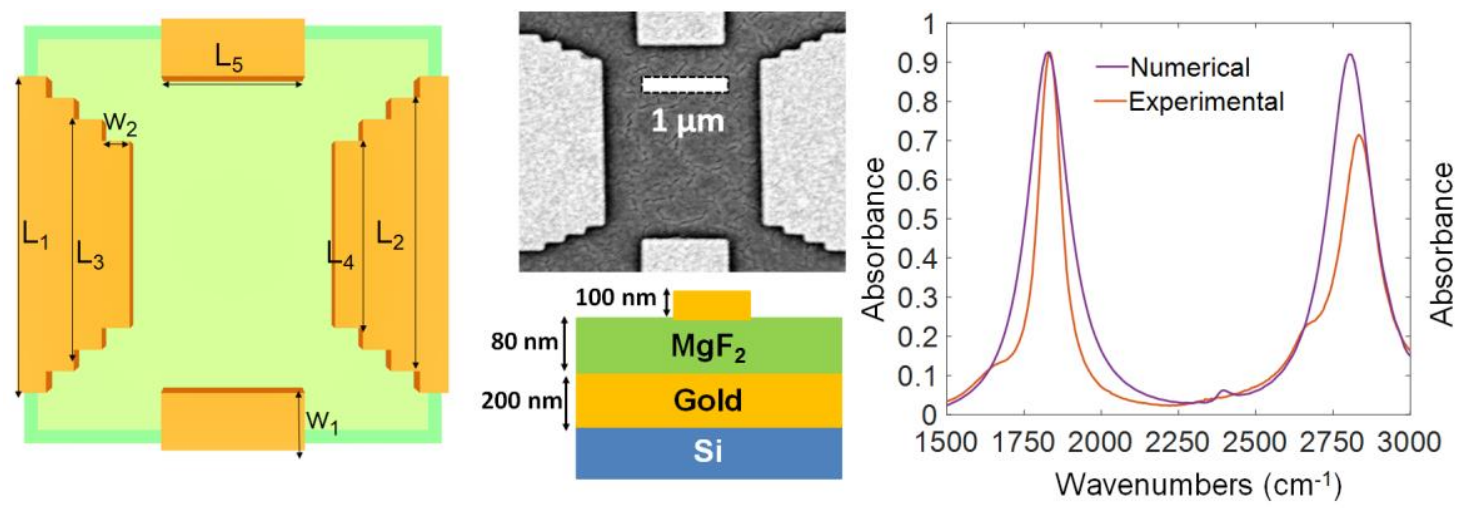

Figure 1. The illustration of top (left) and cross-sectional (middle) view of the PA platform. Middle shows the SEM image of the fabricated PA arrays. Right shows numerical and experimental dual-resonant absorption spectra of the PA under $\mathrm{x}$ polarized illumination source for the mentioned PA size ${ }^{16}$.

Figure 1 shows numerical and experimental absorption spectra of the PA. Numerical and experimental absorption spectra are in well agreement for the first mode at smaller wavenumbers in the terms of resonance frequency and absorption value. For the second mode at higher wavenumber, we obtain nearly the same resonance frequency and lower intensity. The fullwidth at half maximum (FWHM) for experimental results are surprisingly narrower than the simulation results. This effect is still under investigation; however, we think it can be explained by the curved corners of the fabricated structures compared to the sharp edges used in the simulations. We call this PA as a narrowband PA since previous studies on multi-mode multilayer PA's $\mathrm{s}^{3}$ regularly provided considerably broader $\left(167.5 \mathrm{~cm}^{-1}\right) \mathrm{FWHM}$ values in the midIR regime. The PA platform supports two resonance modes; the first mode is observed at around $1830 \mathrm{~cm}^{-1}$ with more than $90 \%$ absorption. The second mode is obtained at $2810 \mathrm{~cm}^{-1}$ with $70 \%$ absorption, while numerical results show 90 
$\%$. This is because the electromagnetic coupling is not strong enough between the vertical and horizontal rods in the unit cell.

\section{RESULTS AND DISCUSSION}

We design the simulation platform identical to the configuration that we take the spectroscopic measurements ${ }^{16}$. Thus, the light illumination has been a plane wave, sent normally on the structures. There is no angle introduced on the IR light illumination, both in measurements and in simulations. From the simulations we know that the structures are polarization dependent, so we applied the same polarization direction that is used in the simulations to the IR illumination in the microscope. We have analyzed the effect of periodicity of PA, the effect of polarization angle and angle of incidence using simulations (Figure 2). As the angle of incidence deviates from normal, the perfect absorption $(\mathrm{A}=1)$ is lost and resonance intensity decreases. Similarly, when the polarization angle is changed from zero to 30 degrees, the perfect absorption is lost. When we increase periodicity, there is no significant change for the first resonance mode, but the second resonance slightly red shifts.

(a)

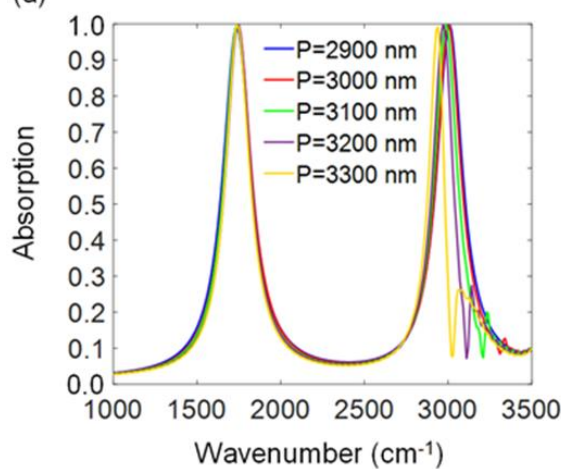

(b)

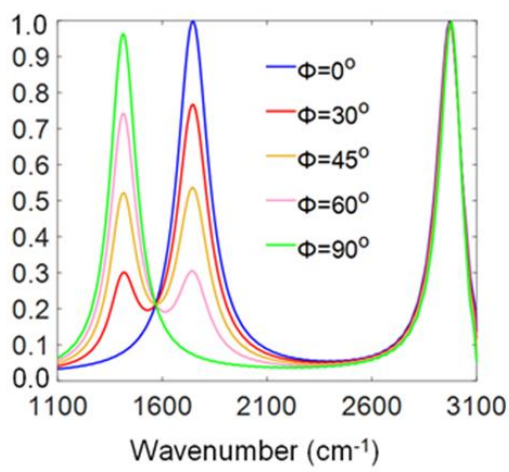

(c)

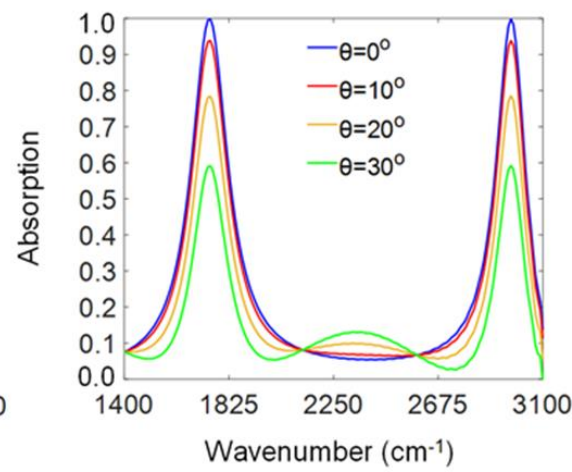

Figure 2. (a) Sweep for periodicity of the structure. $\mathrm{P}$ is same for both $\mathrm{x}$ and $\mathrm{y}$. Effects of the polarization angle $\phi$ (b) and incidence angle $\theta$ (c) on the absorption spectrum of PA. The array periodicity is $3.2 \mu \mathrm{m}^{16}$.

The dielectric spacer has the maximum impact on the perfect absorption as shown in Figure 3. The prior simulation results revealed that the perfect absorption $(\mathrm{A}=1)$ can be achieved at various spacer thickness periodically at certain operation frequencies ${ }^{17}$. When the thickness is low $(<200 \mathrm{~nm})$ strong coupling between the top antenna and ground plate occurs. The thickness of the spacer must be tuned critically to provide destructive interference of light that is directly reflected from the top antenna layer and reflections occurring in the spacer. Any change of the spacer thickness lightly affects the FWHM, but the absorption changes dramatically.

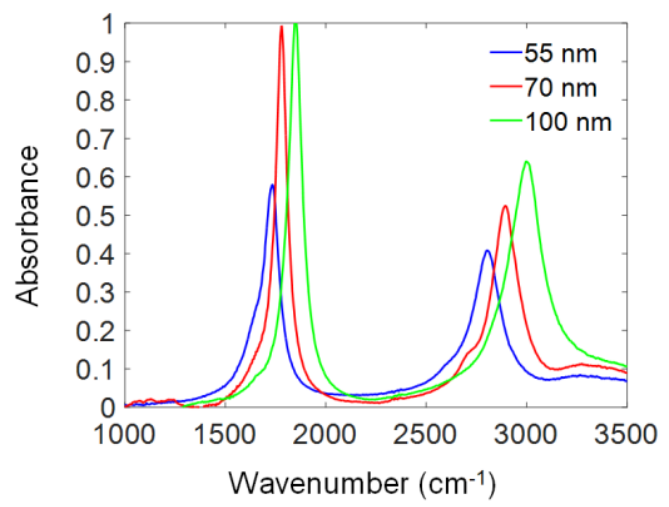

Figure 3. Experimental absorbance spectrum of $\mathrm{PA}$ for different $\mathrm{MgF}_{2}$ thickness. The $\mathrm{MgF}_{2}$ thickness must be tuned carefully to obtain narrow band resonances and near unity absorbance ${ }^{16}$. 
To demonstrate the capacity of the suggested narrow-band PA in surface enhanced infrared absorption spectroscopy, we performed simultaneous detection of two specific and spectrally far vibration modes of a polymeric film experimentally. We coated a $10 \mathrm{~nm}$-thick PMMA film by spin-coating (5000 rpm, $25 \mathrm{~s}$.). Thickness of the PMMA film is characterized using J. A. Woollam Co. VASE ellipsometer. Control measurements are performed on an $\mathrm{Au}$ coated $\mathrm{MgF}_{2}$ film.

No molecular absorption signals are obtained for the same PMMA film thickness on the control measurements. Figure 4 (b) present experimental absorption spectra of the PA platform with $70 \mathrm{~nm}$-thick $\mathrm{MgF}_{2}$ layer after being coated by PMMA film. The PA parameters L1and L5that overlaps with the PMMA absorption peaks are determined as $2400 \mathrm{~nm}$ and $790 \mathrm{~nm}$, respectively. Absorption is more evident when L1increases since the overlap of carboxyl bond with PA resonance is maximum. As it is seen in the Figure 4, when we match plasmonic resonance frequency $\left(1729 \mathrm{~cm}^{-1}\right)$ with the molecular absorption frequency of carbonyl bands, PMMA starts to absorb more energy from nanoantenna which offers the maximum enhanced field available. The carbonyl $\mathrm{v}(\mathrm{C}=\mathrm{O})$ stretch band frequency is observed at $1729 \mathrm{~cm}^{-1}$ and asymmetric methyl $\mathrm{v}(-\mathrm{CH} 3)$ stretch band is observed at $2952 \mathrm{~cm}^{-1}$. The absorption intensity of the carbonyl $(\mathrm{C}=\mathrm{O})$ band on the PA resonance is stronger than the absorption intensity of $-\mathrm{CH}_{3}$ asymmetric band. Finally, we experimentally perform surface enhanced infrared absorption spectroscopy for all PA arrays to show the importance of matching narrow band resonances with molecular absorption bands. Absorption dip for the $\mathrm{C}=\mathrm{O}$ stretch band (around $1729 \mathrm{~cm}^{-1}$ ) can be clearly seen in Figure 4. When L5 is equal to $790 \mathrm{~nm}$, we obtain the strongest absorption signal of the $-\mathrm{CH}_{3}$ asymmetric band stretch. The experimental spectra of PMMA coated PA is subtracted from the uncoated PA's experimental spectra for the $\mathrm{C}=\mathrm{O}$ band (red line) and the $-\mathrm{CH}_{3}$ asymmetric band (blue line), respectively. After PMMA binding no red-shift is observed on the PA resonance for the first mode, thus we applied direct subtraction without correcting the shift with a polynomial fitting. In order to reliably calculate the absorption difference in the presence of the spectral shifts for the second mode, we employ the fitting procedure which shifts the bare response and performs a baseline fitting such that the PA absorption before PMMA coating fairly overlaps with one after PMMA coating. In Figure 4, when the resonance frequency of the PA is exactly matched with absorption frequency of the $\mathrm{C}=\mathrm{O}$ band, absorption difference, and thus the sensitivity reaches its maximum value. Absorption difference decreases when the PA resonance is even slightly off from the carbonyl absorption frequency. The absorption difference varies between $13 \%$ and $33 \%$ of the PA resonance intensity. Even when the PA resonance is very off from the carbonyl absorption frequency, we still see a strong signal obtained from an only $10 \mathrm{~nm}$-thick PMMA film. The coupling between the plasmonic and PMMA vibrational modes results in spectral red-shift in the $\mathrm{C}=\mathrm{O}$ absorption dip from their usual spectral position at $1729 \mathrm{~cm}^{-1}$, as shown by the vertical red line in Fig. 4(b). When L5= $790 \mathrm{~nm}, 1.5 \%$ absorption difference is obtained for the $-\mathrm{CH}_{3}$ asymmetric band stretch at $2952 \mathrm{~cm}^{-1}$. For other lengths of L5, absorption differences at the $-\mathrm{CH}_{3}$ asymmetric band stretch frequency is not significant.
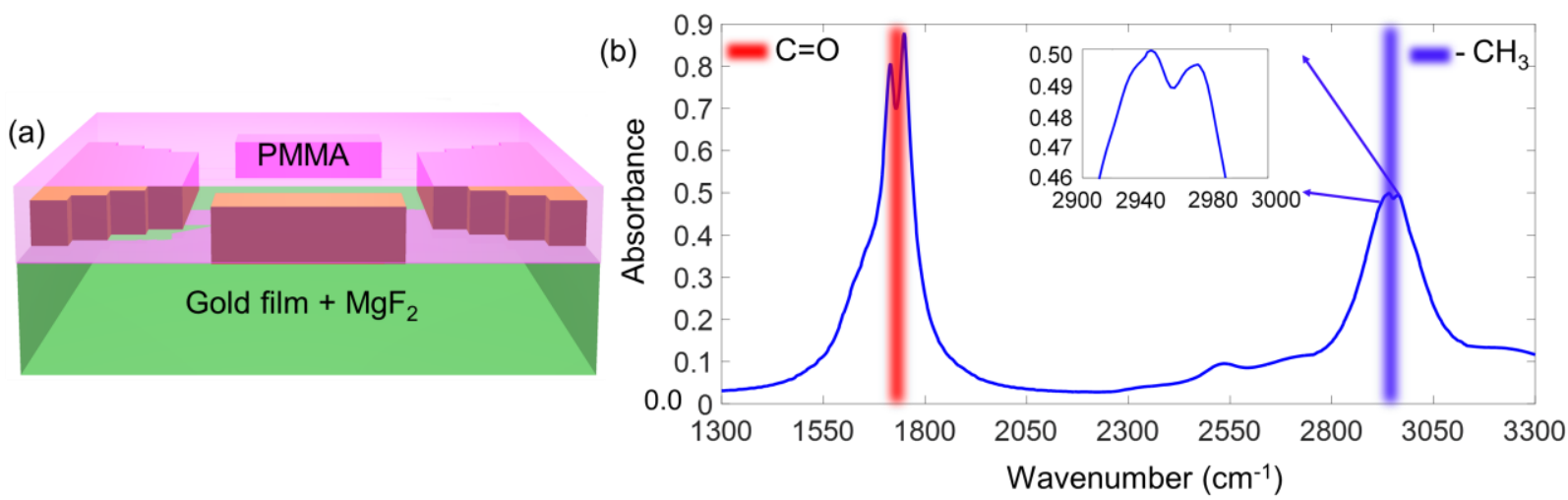

Figure 4. (a) $10 \mathrm{~nm}$-thick PMMA film is spin-coated on top of the nanoantennas. (b) Surface enhanced infrared absorption spectroscopy of a $10 \mathrm{~nm}$-thick PMMA film on fabricated narrow band perfect absorbers when $\mathrm{L} 1=2400 \mathrm{~nm}$ and $\mathrm{L} 5=790 \mathrm{~nm}$. Experimental difference spectroscopy for the $\mathrm{C}=\mathrm{O}$ band at the first resonance mode - $\mathrm{CH} 3$ asymmetric band at the second resonance mode are calculated. The absorption is $33 \%$ of the PA resonance intensity for the $\mathrm{C}=\mathrm{O}$ band and $1.5 \%$ for the $-\mathrm{CH}_{3}$ asymmetric band ${ }^{16}$. 


\section{CONCLUSION}

In conclusion ${ }^{16}$, we experimentally presented a narrow band $\left(85 \mathrm{~cm}^{-1}\right)$ multi-resonant perfect absorber operating in the mid-IR range for the first time and demonstrated its usage for surface enhanced infrared absorption spectroscopy. Firstly, we performed theoretical analysis to investigate the physical origin of the dual-resonant behavior with strong near-field intensity enhancement distributions. Then, we obtained a fine-tuning mechanism to control the spectral response of the PAs through the geometrical parameters. Finally, we fabricated the numerically designated PAs and observed that the fabricated systems show similar optical responses compared to the calculations. In addition, we realized that the fabricated PAs show narrower resonance bands compared to the simulations or monolayer plasmonic nanoantennas. We also used the fabricated PAs to experimentally detect molecular fingerprints obtained from a $10 \mathrm{~nm}$-thick PMMA film. The SEIRA spectroscopy resulted in 33\% intensity change on the PA resonance, which shows the sensitivity improvement obtained from narrow band resonances in comparison to monolayer plasmonic antennas. Fine tuning of the narrow-band PAs with high quality factor resonances allowed us to exactly match the relevant vibrational molecular modes. Thus, the presented narrow band multi-mode PA system is a promising probe to study very thin protein-lipid combined systems, such as live cell-membranes.

*saksu@ku.edu.tr; phone +90 212338 1568; serapaksu.com

\section{REFERENCES}

[1] Atwater H.A. and Polman A. "Plasmonics for improved photovoltaic devices," Nat. Mater. 9(3), 205-213 (2010).

[2] Liu, N. et al. "Infrared Perfect Absorber and Its Application As Plasmonic Sensor," Nano Lett. 10(7), 23422348 (2010).

[3] Chen, K.et al. "Dual-Band perfect absorber for multispectral plasmon-enhanced infrared spectroscopy," ACS Nano 6, 9, 7998-8006, (2012).

[4] Chen, S. et al. "Ultra-Narrow band Mid-Infrared perfect absorber based on hybrid dielectric metasurface," Nanomaterials 9, 1350, (2019).

[5] Adato R. et al "Engineering mid-infrared nanoantennas for surface enhanced infrared absorption spectroscopy, Mater. Today 18 (2015) 436-446, https://doi.org/10.1016/j.mattod.2015.03.001.

[6] Aksu, S et al. "Plasmonically Enhanced Vibrational Biospectroscopy Using Low-Cost Infrared Antenna Arrays by Nanostencil Lithography" Adv. Opt. Mater. 1 (2013) 798-803, https://doi.org/10.1002/adom.201300133.

[7] Brown, L.V. " Fan-shaped gold nanoantennas above reflective substrates for surface-enhanced infrared absorption (SEIRA), Nano Lett. 15 (2015) 1272-1280, https://doi.org/10.1021/nl504455s.

[8] Osawa, M et al. "Surface-enhanced infrared spectroscopy: the origin of the absorption enhancement and band selection rule in the infrared spectra of molecules adsorbed on fine metal particles," Appl. Spectrosc. 47 (1993) 1497-1502, https://doi.org/10.1366/0003702934067478.

[9] Landy, N.I. “ Perfect metamaterial absorber,” Phys. Rev. Lett. 100 (2008) 207402 , https://doi.org/10.1103/PhysRevLett.100.207402.

[10] Aslan, E.et al. "Polarization insensitive plasmonic perfect absorber with coupled antisymmetric nanorod array", Sens. Actuators, B, 243 (2017) 617-625, https://doi.org/10.1016/j.snb.2016.12.030.

[11] Mason, J.A. et al. "Strong coupling of molecular and mid-infrared perfect absorber resonances", IEEE Photonics Technol. Lett. 24 (2012) 31-33, https://10.1109/LPT.2011.2171942 
[12] Wu, C. et al. "Fano-resonant asymmetric metamaterials for ultrasensitive spectroscopy and identification of molecular monolayers", Nat. Mater. 11 (2012) 69-75, https://doi.org/10.1038/nmat3161.

[13] W. Klöpffer, Introduction to polymer spectroscopy Volume VII, Springer Science \& Business Media, 2012.

[14]E. D. Palik, Handbook of optical constants of solids Volume III, Academic Press, 1998.

[15] M. J. Dodge, Refractive properties of magnesium fluoride, Appl. Opt. 23 (1984) 1980-1985, https://doi.org/10.1364/AO.23.001980.

[16] Korkmaz, S. "Mid-infrared narrow band plasmonic perfect absorber for vibrational spectroscopy "Sensors and Actuators A 301, 111757, (2020).

[17] Alaee, R et al.” Theory of metasurface based perfect absorbers,” J. Phys. D: Appl. Phys. 50, 503002, (2017) https://doi.org/10.1088/1361-6463/aa94a8. 